\title{
Radiative accelerations in stellar atmospheres
}

\author{
A. Hui-Bon-Hoa ${ }^{1}$, F. LeBlanc ${ }^{2}$, P. H. Hauschildt ${ }^{3}$, and E. Baron ${ }^{4}$ \\ 1 Laboratoire d'Astrophysique Extragalactique et de Cosmologie, CNRS-(UMR 8631), Observatoire de Paris, \\ Université Paris 7, DAEC, Observatoire de Meudon, 92195 Meudon Cedex, France \\ 2 Département de Physique et d'Astronomie, Université de Moncton, Moncton, N.-B., Canada E1A 3E9 \\ 3 Department of Physics and Astronomy, University of Georgia, Athens, GA 30602-2451, USA \\ 4 Department of Physics and Astronomy, University of Oklahoma, 440 W. Brooks, Room 131, Norman, OK, \\ 73019-0225 USA
}

Received 16 August 2001 / Accepted 22 October 2001

\begin{abstract}
We present calculations of radiative accelerations obtained with the general purpose model atmosphere code PHOENIX. The accelerations are computed simultaneously for all the elements $\mathrm{He}-\mathrm{Ga} ; \mathrm{Kr}-\mathrm{Nb} ; \mathrm{Ba} ; \mathrm{La}$, using the opacity sampling method. The calculations are mainly performed with the LTE approximation. Tests are made to evaluate the influence of non-LTE effects and the validity of the radiative flux obtained through the diffusion approximation. It is shown that the abundances supported in the atmospheres of HgMn and He-weak stars are generally consistent with those observed.
\end{abstract}

Key words. diffusion - stars: atmospheres - stars: chemically peculiar - stars: abundances

\section{Introduction}

The Chemically Peculiar (CP) stars have abundances very different from what can be expected from classical stellar models. Best known are those lying on the Main Sequence (e.g. Preston 1974), but such objects are also present on the Horizontal Branch (Greenstein \& Sargent 1974; Glaspey et al. 1989) and on the white dwarf cooling sequence (e.g. Vauclair et al. 1979).

It is now well established that the vast majority of the abundance anomalies observed in CP stars are due to microscopic diffusion (Michaud 1970; Greenstein et al. 1967; Michaud et al. 1983; Schatzman 1958). As time goes by, the particles migrate according to their photoabsorption properties and the abundances of each element stratify. In each layer of the star, the particles move according to a diffusion velocity that involves a term describing the momentum transfer between the radiation field and the various chemical species: the radiative acceleration. Thus, the first step of a diffusion study is the calculation of radiative accelerations.

In the past, most computations of radiative accelerations were performed in optically thick media, where the radiative flux can be expressed by an analytical formula (the so-called diffusion approximation, or Milne 1927 flux). This approximation is therefore only suitable for stars with thick enough a superficial mixing layer (e.g.

Send offprint requests to: A. Hui-Bon-Hoa, e-mail: alain.hui@obspm.fr
Am stars, Richer et al. 2000), diffusion then occurring in the envelope. In such cases, several methods of calculation have been developed, differing mainly by the estimation of the "background opacity" (i.e. the opacity due to all other sources but the atomic transition under consideration). For instance, Borsenberger et al. (1979) used a simple function scaled with the Rosseland opacity. In Gonzalez et al. (1995b, hereafter GLAM), the background opacity is approximated by an average calculated in 4000 frequency intervals. The radiative accelerations on CNO (Gonzalez et al. 1995a), Fe (LeBlanc \& Michaud 1995), and several other elements (LeBlanc et al. 2000, hereafter LMR2000) have been calculated this way. More accurate radiative accelerations are obtained with the sampling method, which consists of direct integration on a given frequency grid. It has been used by Seaton (1997) and LMR2000 with Opacity Project atomic data (Seaton 1987; Seaton et al. 1992; Cunto et al. 1993), whereas Richer et al. (1998) used OPAL opacities (Rogers \& Iglesias 1992a,b; Iglesias \& Rogers 1995, 1996). Alecian (1985) and Alecian \& Artru (1990) introduced a method that parametrizes the radiative accelerations due to bound-bound transitions, in which two parameters express the intrinsic photoabsorption properties of the ion and the plasma conditions. They are both computed with atomic data. No frequency integration is needed so that it saves much computing time, the results being in good agreement compared to the detailed calculations (differences less than $25 \%$, Alecian et al. 1993). However, this method does not consider the effect 
of line blending and the composition of the medium should not be too different from that for which the parameters have been computed.

For hotter stars (e.g. Ap stars), diffusion occurs in the atmosphere and the radiative transfer equation has to be solved explicitly. The case of white dwarfs has been recently addressed by Dreizler (1999), who computed detailed radiative accelerations for several elements with the opacity sampling method, thus considering the mutual influence between the various chemical species. Concerning the Main Sequence, the few studies performed up to now used codes that considered only one element at a time and the radiative flux did not include any metallic line opacity (e.g. Borsenberger et al. 1979; Alecian \& Michaud 1981; Alecian \& Vauclair 1981; Alecian \& Artru 1987; Hui-Bon-Hoa et al. 1996). The effects of blends were thus not taken into account. The results were nevertheless in good qualitative agreement with the observed anomalies. The case of aluminium (Hui-Bon-Hoa et al. 1996), with radiative accelerations very near gravity, showed the need for a greater accuracy in these kinds of computations.

We present here radiative accelerations obtained with a new code dedicated to the study of diffusion in stellar atmospheres, which has been recently used in the case of blue Horizontal-Branch stars (Hui-Bon-Hoa et al. 2000). A basic assumption for this study is a static medium, which is justified because hot CP stars are expected to have hydrodynamically stable atmospheres. Indeed, they are supposed to have very thin superficial convective layers and the large scale motions are prevented by their slow rotation. The effect of possible mass-loss is neglected.

In Sect. 2, we detail the implementation of the computation of radiative accelerations. Then, in Sect. 3, we will present our results. We will also show the limits of the so-called diffusion approximation where the radiation flux is obtained through an analytical expression and test the effect of departures from LTE. Comparisons of our results with the anomalies observed in Main Sequence CP stars (HgMn; He-weak stars) are shown in Sect. 4.

\section{Computation of radiative accelerations}

Model atmosphere codes are natural starting points for the computation of radiative accelerations since the quantities needed are the same as those required for the radiative equilibrium. The treatment of the radiative transfer using the opacity sampling method is very convenient for the calculation of radiative accelerations (e.g. LMR2000) and we have thus chosen to implement diffusion in the general purpose stellar atmosphere code PHOENIX (Hauschildt et al. 1996; Hauschildt et al. 1997a,b; Baron \& Hauschildt 1998; Hauschildt \& Baron 1999; Barman et al. 2000). An extensive description of PHOENIX can be found in the above-mentioned papers (and references therein) so that we will focus here mainly on the implementation of the calculation of radiative accelerations.
The radiative accelerations $\left(g_{\mathrm{rad}}\right)$ express the momentum transfer between the radiation field and the various ions and can be written as follows:

$g_{\mathrm{rad}}(A)=\frac{4 \pi}{c} \frac{1}{X_{A}} \int_{0}^{\infty} \kappa_{\lambda}(A) H_{\lambda} \mathrm{d} \lambda$

where $X_{A}$ is the mass fraction of ion $A, \kappa_{\lambda}(A)$ the monochromatic opacity of this species, and $H_{\lambda}$ the monochromatic (Eddington) radiation flux. For each ion, the radiative accelerations obtained through bound-bound and bound-free absorptions are calculated separately since for a species in the ionization stage $i$, the radiative acceleration gained by photoionization will benefit to ion $i+1$. We thus consider separately the line and continuum opacities.

The monochromatic opacities are calculated by PHOENIX during each iteration according to the chemical composition and the atmospheric structure. $H_{\lambda}$ is obtained through the detailed resolution of the transfer equation, done with the opacity sampling method (for a discussion about radiative accelerations obtained with the sampling method see LMR2000). For test purposes, we have also implemented the computation of the flux according to the so-called diffusion approximation, valid in optically thick media, and obtained through an analytical expression (Milne 1927). With this formulation, widely used in stellar envelopes, one can avoid solving the transfer equation. The radiative flux is then given by (e.g. Eq. (2-91) of Mihalas 1978)

$H_{\lambda}=-\frac{1}{3} \frac{1}{\kappa_{\lambda}} \frac{\partial B_{\lambda}}{\partial T} \frac{\partial T}{\partial r}$

where $B_{\lambda}$ is the Planck function, $T$ the temperature, and $r$ the radius. When radiative equilibrium is assumed, the temperature gradient can be derived using the definition of the Rosseland mean:

$\frac{\partial T}{\partial r}=-\frac{3}{16} \kappa_{\mathrm{R}} \frac{T_{\mathrm{eff}}^{4}}{T^{3}} \frac{R^{2}}{r^{2}}$

and the flux expression becomes

$H_{\lambda}=\frac{1}{16} \frac{\kappa_{\mathrm{R}}}{\kappa_{\lambda}} \frac{\partial B_{\lambda}}{\partial T} \frac{T_{\mathrm{eff}}^{4}}{T^{3}} \frac{R^{2}}{r^{2}}$

$\kappa_{\mathrm{R}}$ is the Rosseland opacity, $T_{\text {eff }}$ the effective temperature and $R$ the stellar radius.

The diffusion velocity for an ion of a trace element (Burgers 1960) can be approximated by

$$
\begin{aligned}
V_{i}= & D_{i}\left\{-\frac{\partial \ln c_{i}}{\partial r}+\frac{m_{i}}{k T}\left[g_{\mathrm{rad}}^{i}-\left(\frac{2 A_{i}-Z_{i}-1}{2 A_{i}}\right) g\right]\right. \\
& \left.+\left(3.45 Z_{i}^{2}-0.82\right) \frac{\partial \ln T}{\partial r}\right\}
\end{aligned}
$$

In this expression the drag terms between the different kinds of particles are neglected since we suppose that we have trace elements. In this equation, $m_{i}$ is the mass, $A_{i}$ the atomic mass, $Z_{i}$ the charge, $g$ is the local gravitational acceleration and $g_{\mathrm{rad}}^{i}$ the radiative acceleration. We then define the effective acceleration $\left(g_{\text {eff }}\right)$ as

$g_{\mathrm{eff}}^{i}=g_{\mathrm{rad}}^{i}-\left(\frac{2 A_{i}-Z_{i}-1}{2 A_{i}}\right) g$ 
that is, the sum of the radiative, gravitationnal and electrical forces acting on ion $i$.

As time goes by, a particle in an initial ionization stage $i$ can ionize or recombine, thereby transferring part of the momentum acquired in stage $i$ to adjacent ones. This momentum sharing among the various ionization stages of an element is called the redistribution effect (Montmerle \& Michaud 1976). To know the part of the momentum given to other ionization stages, we have to estimate the ionization/recombination rates for each energy state of the ions. For this, GLAM considered the principal quantum number of the level to decide whether the momentum should be kept in the initial ionization stage $i$ or given to stage $i+1$. Their calculation on CNO showed that the recombination rates were always much smaller than the collisional or ionization ones so that no momentum is granted to ion $i-1$. However, this approach is too complex to be implemented presently in the code, so we rather turn to the method of Montmerle \& Michaud (1976), which uses rates averaged over the energy levels of each ion. The ionization/recombination rates are computed with the method of Hui-Bon-Hoa et al. (1996), using the continuous opacities of each ion, in LTE (Local Thermal Equilibrium) as well as in NLTE (non-LTE) mode. Since only the radiative processes are considered, the redistribution effect may be underestimated, but the contribution of collisional processes is negligible in the upper part of the atmospheres (at least in the case of aluminium, Hui-Bon-Hoa et al. 1996), where redistribution is the most efficient.

Since we are interested in the total radiative acceleration acting on a given element, one has to estimate the mean radiative acceleration. In the present case, we use the following weighted mean:

$g_{\mathrm{rad}}=\frac{\sum_{i} N_{i} D_{i} g_{\mathrm{rad}}^{i}}{\sum_{i} N_{i} D_{i}}$.

It reflects the expression of the total diffusion velocity, when the weighted average is done using the populations. $N_{i}$ is the relative population of ion $i$ and $D_{i}$ its diffusion coefficient. We used a formula coming from expressions $(9.81,1)$ and $(10.31,11)$ of Chapman \& Cowling $(1970)$ for the diffusion coefficients of the neutral species, with values of electric polarisability taken from the CRC handbook of Phys. and Chem. (1997). As for the charged ions, we used expression (2.22) of Aller \& Chapman (1960) (for details about the diffusion coefficients, see GLAM, Appendix B). We only consider a single diffusion coefficient per ionization stage, thus neglecting the variation from one excitation state to another (see LeBlanc \& Michaud 1993 for a discussion on the dependence of diffusion coefficient vs. excitation state).

It should be noted that in practice, we adapted for our calculations of the total radiative acceleration parts of the code used in previous diffusion studies in atmospheres (Alecian \& Michaud 1981; Alecian \& Vauclair 1981; Alecian \& Artru 1987; Hui-Bon-Hoa et al. 1996).

\subsection{Computations assuming LTE}

The line opacities are calculated with the atomic data of Kurucz' (1994) linelist for the elements included in PHOENIX, namely $\mathrm{He}-\mathrm{Ga}, \mathrm{Kr}-\mathrm{Nb}, \mathrm{Ba}$, and La. The lines are selected according to the ratio $\chi_{\mathrm{lc}} / \kappa_{\mathrm{c}}$ between the extinction at the line center $\chi_{\mathrm{lc}}$ and the local continuous opacity $\kappa_{\mathrm{c}}$ (Hauschildt et al. 1999), and to the wavelength mesh. We have set the threshold value at $10^{-5}$. We tried to find the best compromise between computing time and accuracy of the results by adjusting the wavelength step. When increasing the number of grid points (refining the mesh), the amount of lines selected is roughly the same (around 2 million for the $12000 \mathrm{~K}$ model with a threshold value of $10^{-5}$ ) and the variations of the radiative accelerations come from the way the lines are sampled. The radiative accelerations through line absorptions for some ions are very sensitive to the choice of the wavelength grid, in particular in the most superficial layers (for instance, about $1 \mathrm{dex}$ for $\mathrm{Ne}$ when the step in the UV and the visible varies from 0.4 to $0.02 \AA$ ), where the lines are the least broadened. The radiative accelerations converge well even in the uppermost layers for a 500000 points grid, with a step of $20 \mathrm{~m} \AA$ between $350 \AA$ and $1 \mu \mathrm{m}$, where most of the flux is emitted (given the effective temperatures of the models), and where the majority of the contributing lines are. We actually used this grid in the calculations shown in Sect. 3.1.2. We noticed that the choice of the wavelength grid has much more influence on the radiative accelerations than the opacity threshold: the results with a threshold at $10^{-3}$ are almost the same as those using a ratio of $10^{-5}$. The largest differences amount to less than $10 \%$ in the worst cases. The microturbulence velocity has been set to zero, since CP stars' atmospheres are assumed to be hydrodynamically stable.

The radiative accelerations due to bound-free transitions were computed using the "hydrogenic" $\nu^{-3}$ power law cross-sections implemented for the NLTE ions, which are scaled to the results of Reilman \& Manson (1979), as described in Hauschildt et al. (1996), instead of the data of Mathisen (1984), which are used in the building of the atmospheric structure. The hydrogenic cross-sections are less accurate but are available for far more ions and levels within each ion. Also, for an ionizing transition, part of the momentum is taken away by the ejected electron. Apart from the usual expression of Sommerfeld \& Schur (1930) for the $1 s$ state of hydrogen (H1S), very little is known about the fraction of the momentum kept by the ion, $f_{\text {ion }}$. Detailed calculations have only been done for hydrogenic ions (Massacrier 1996) and lithium (Massacrier \& El-Murr 1996). Owing to the lack of accurate data, the H1S expression of $f_{\text {ion }}$ is often adopted for all the elements, the results of Richer et al. (1997) showing that this approximation gives reasonably accurate results, at least in the case of lithium. The results shown here assume no correction factor, i.e. $f_{\text {ion }}=1$, except for test purposes. 
Table 1. NLTE species of PHOENIX (version 10). The first column lists the elements by increasing atomic number, the other the number of levels/transitions ("primary lines", see text) for each corresponding ion.

\begin{tabular}{|c|c|c|c|c|c|c|c|}
\hline Element & I & II & III & IV & $\mathrm{V}$ & VI & VII \\
\hline $\mathrm{H}$ & $80 / 3160$ & & & & & & \\
\hline $\mathrm{He}$ & $19 / 37$ & $10 / 45$ & & & & & \\
\hline $\mathrm{Li}$ & $57 / 333$ & $55 / 124$ & & & & & \\
\hline $\mathrm{C}$ & $228 / 1387$ & $85 / 336$ & $79 / 365$ & $35 / 171$ & & & \\
\hline $\mathrm{N}$ & $252 / 2313$ & $152 / 1110$ & $87 / 266$ & $80 / 388$ & $39 / 206$ & $15 / 23$ & \\
\hline $\mathrm{O}$ & $36 / 66$ & $171 / 1304$ & $137 / 765$ & $134 / 415$ & $97 / 452$ & $39 / 196$ & \\
\hline $\mathrm{Ne}$ & $26 / 37$ & & & & & & \\
\hline $\mathrm{Na}$ & $53 / 142$ & $35 / 171$ & $69 / 353$ & $46 / 110$ & $64 / 187$ & $102 / 375$ & \\
\hline $\mathrm{Mg}$ & $273 / 835$ & $72 / 340$ & $91 / 656$ & $54 / 169$ & $53 / 133$ & $78 / 180$ & \\
\hline $\mathrm{Al}$ & $111 / 250$ & $188 / 1674$ & $58 / 297$ & $31 / 142$ & $49 / 77$ & $40 / 93$ & \\
\hline $\mathrm{Si}$ & $329 / 1871$ & $93 / 436$ & $155 / 1027$ & $52 / 292$ & $35 / 125$ & $36 / 49$ & \\
\hline $\mathrm{P}$ & $229 / 903$ & $89 / 760$ & $51 / 145$ & $50 / 174$ & $40 / 204$ & $10 / 9$ & \\
\hline S & $146 / 439$ & $84 / 444$ & $41 / 170$ & $28 / 50$ & $19 / 41$ & $31 / 144$ & \\
\hline K & $73 / 210$ & $22 / 66$ & $38 / 178$ & $24 / 57$ & $29 / 75$ & & \\
\hline $\mathrm{Ca}$ & $194 / 1029$ & $87 / 455$ & $150 / 1661$ & $67 / 122$ & $39 / 91$ & $23 / 37$ & $26 / 59$ \\
\hline $\mathrm{Ti}$ & $395 / 5279$ & $204 / 2399$ & & & & & \\
\hline $\mathrm{Fe}$ & $494 / 6903$ & $617 / 13675$ & $566 / 9721$ & $243 / 2592$ & $132 / 961$ & $87 / 551$ & \\
\hline $\mathrm{Co}$ & $316 / 4428$ & $255 / 2725$ & $213 / 2248$ & & & & \\
\hline $\mathrm{Ni}$ & $153 / 1690$ & $429 / 7445$ & $259 / 3672$ & $189 / 1845$ & $245 / 2638$ & $246 / 2868$ & \\
\hline $\mathrm{Rb}$ & $29 / 76$ & & & & & & \\
\hline $\mathrm{Sr}$ & $52 / 74$ & $32 / 90$ & & & & & \\
\hline Cs & $38 / 75$ & & & & & & \\
\hline $\mathrm{Ba}$ & $76 / 114$ & $51 / 121$ & & & & & \\
\hline
\end{tabular}

\subsection{Non-LTE calculations}

Departures from LTE can be considered for 90 atoms in PHOENIX (version 10). A summary of the data is given in Table 1. Let us recall that the model atoms are built using the linelist of Kurucz (1994). The "primary" lines, used in the rate equations, are selected as their $g f$-value is greater than a threshold set at $10^{-3}$. All the levels concerned by these transitions are included in the model atom. Weaker transitions ("secondary" lines) thus involve at least one level not treated explicitly in the rate equations, for which the ground state departure coefficients are applied (see Hauschildt et al. 1996 for more details). Complete NLTE effects therefore concern only the primary lines but we also compute radiative accelerations from the secondary lines for an estimation of their contribution. In NLTE mode, in addition to the user specified grid (see above), wavelength points are set according to the primary NLTE line central wavelengths with additional points at given distances of the line cores if needed. The grid is then modified to take the NLTE points into account without increasing too much the number of grid points.

\section{Radiative accelerations for Main Sequence CP stars}

Diffusion occurs in stellar atmospheres only if the macroscopic motions are weak enough. On the Main Sequence, this condition is fulfilled for $T_{\text {eff }}$ between approximately 10000 and 30000 K. For the hottest stars, mass-loss must be considered and the assumption of a static medium is not valid. We thus decided to compute radiative accelerations in two stellar models of $12000 \mathrm{~K}$ (typical HgMn star) and $18000 \mathrm{~K}$ models (He-weak star). As we do not consider any magnetic field effect in our models, the case of the magnetic CP stars cannot be addressed.

The model atmospheres were built assuming static medium and plane-parallel symmetry, approximations which are valid for the present temperature range (Aufdenberg et al. 1998, 1999). The computations are made considering solar abundances (Grevesse \& Noels 1993) throughout the atmosphere.

In the following, we present our radiative accelerations results.

\section{1. $L T E$ radiative accelerations}

\subsubsection{Using the diffusion approximation for the radiative flux}

As the diffusion approximation is widely used in the radiative acceleration computations in stellar interiors, we wanted to test the validity of this approximation in the atmosphere. The computation was made for a $12000 \mathrm{~K}$ model.

Though the ratios of the fluxes integrated over the frequencies are very close to unity whatever the optical depth, the radiative accelerations for individual ions have a different behaviour. In the deep layers, the results from the detailed calculation and the approximation are very 


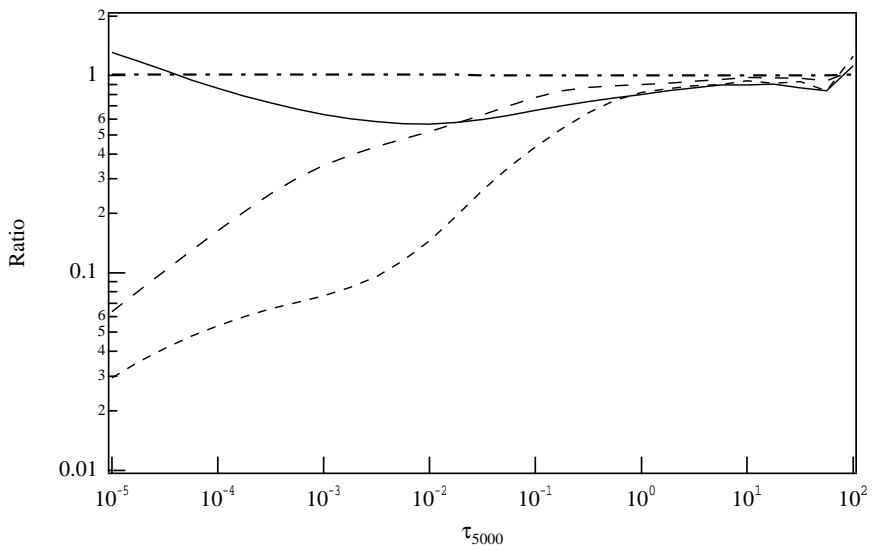

Fig. 1. Ratios of radiative accelerations obtained with the diffusion approximation over those with detailed radiative transfer against optical depth for the ions $(12000 \mathrm{~K}$, $\log g=4$ model): O (short-dashed line); $\mathrm{Na}$ (solid line); $\mathrm{Fe}$ (long-dashed line). The ratio of the integrated fluxes is shown by the thick dot-dash line.

similar, as expected in these optically thick media. In the optically thin part of the atmosphere, the radiative accelerations obtained with the diffusion approximation are underestimated for most elements (Fig. 1). For a few others, they can be more than twice enhanced. The reason is that the integrated fluxes are approximately equal whatever the layer but their wavelength distributions are different in the outermost layers, the detailed flux being stronger at shorter wavelengths (see Fig. 2), as the effect on the radiative accelerations depends on the opacity distribution of each element. This shows the need for a detailed treatment of the radiative transfer in the computation of radiative accelerations in the atmosphere.

\subsubsection{Using the flux from the detailed resolution of the transfer equation}

The details of the individual contributions to the radiative accelerations are shown for Si and Fe (Fig. 3), in the case of the $12000 \mathrm{~K}$ model, the contribution of a given ion $i$ being defined by

$$
\frac{N_{i} D_{i} g_{\mathrm{rad}}^{i}}{\sum_{j} N_{j} D_{j}}
$$

As for the other elements concerned by the present calculation, the total radiative acceleration is dominated by the line contribution. Let us remark that the contribution of the continuum of SiII is significant around $\tau_{5000} \approx 10^{-2}$. One exception is $\mathrm{Li}$ in the $18000 \mathrm{~K}$ model whose radiative acceleration is due mainly to the photoionization of the neutral ion.

If we take into account that part of the momentum acquired through photoionization is granted to the ejected electron, i.e. $f_{\text {ion }} \neq 1$ (the H1S expression), the radiative accelerations from bound-free transitions are slightly reduced in comparison to the case where this effect is neglected, by typically $5 \%$ in the upper layers and up to $30 \%$

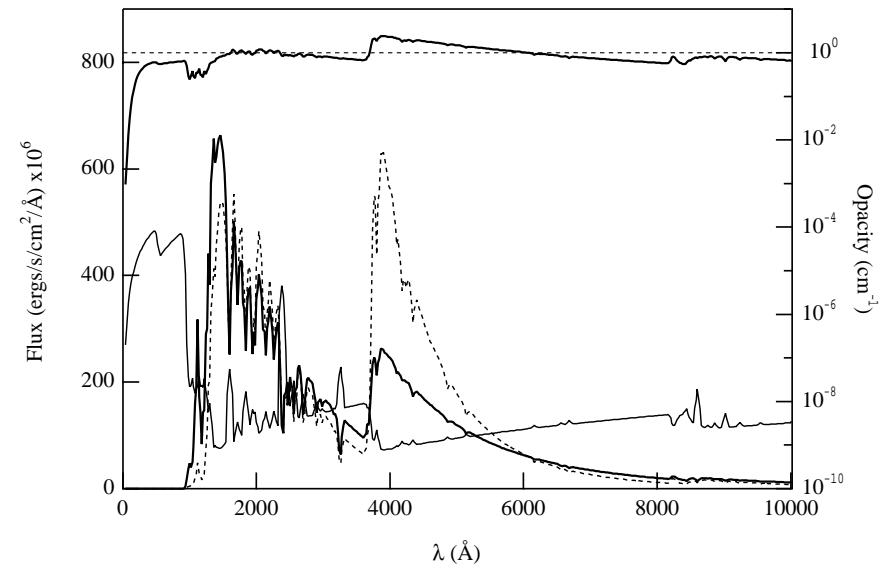

Fig. 2. Comparison of the fluxes (left ordinate axis), for the $12000 \mathrm{~K}, \log g=4$ model, at $\tau_{5000}=10^{-1}$ : obtained with detailed radiative transfer (thick solid line); with the diffusion approximation (dotted line). The ratio of the two is shown in the upper part of the panel (right ordinate axis, the ratio of 1 being shown by the dotted line). At the bottom is drawn the total opacity (thin solid line, right ordinate axis).

at the bottom of the atmosphere (for the $12000 \mathrm{~K}$ model). Since the line contributions dominate the radiative accelerations for most elements, the approximation made for the value of $f_{\text {ion }}$ has no crucial influence on the total radiative accelerations. Anyway, these corrections are much smaller than the differences between the approximation used here and detailed photoionization cross-sections (e.g. Opacity Project data, Alecian 1994; Hui-Bon-Hoa et al. 1996).

Figure 5 displays the radiative accelerations for the $12000 \mathrm{~K}$ and $18000 \mathrm{~K}$ models obtained using the detailed radiative flux. Both the total radiative accelerations before and after applying the redistribution correction are shown. Due to the absence of atomic data, there is no radiative acceleration for $\mathrm{Kr}$ (element not considered in Kurucz' linelist). The same reason accounts for the drop of radiative acceleration at the bottom of the atmosphere for some of the heaviest elements $(\mathrm{Cu}, \mathrm{Ga}, \mathrm{Rb}, \mathrm{Sr}, \mathrm{Y}, \mathrm{Zr}$, $\mathrm{Nb}, \mathrm{Ba}$, and $\mathrm{La}$ ), where the elements are in multicharged ionization stages. Their radiative accelerations can thus be strongly underestimated for layers below $\tau_{5000} \approx 1$ (see for instance the contribution of GaIII and GaIV in Alecian \& Artru 1987).

Redistribution of the momentum has significant effects on several elements (C, Mg, Al, Si, P, S, Fe, and $\mathrm{Ni}$ in the $12000 \mathrm{~K}$ model; CNO, S, and Ca in the $18000 \mathrm{~K}$ model). Moreover, it changes the balance between radiative accelerations and gravity in the upper parts of the atmosphere for $\mathrm{C}, \mathrm{Mg}, \mathrm{Al}, \mathrm{Si}, \mathrm{S}$ in the $12000 \mathrm{~K}$ model, and for CNO in the $18000 \mathrm{~K}$ model. The major effect of redistribution is due to the momentum transfer between neutral and first ionized stages since the diffusion coefficients are very different (about 100 times larger for the neutral for most elements), unless the contribution of the neutral is negligible. Elements for which the redistribution effect is strong have 

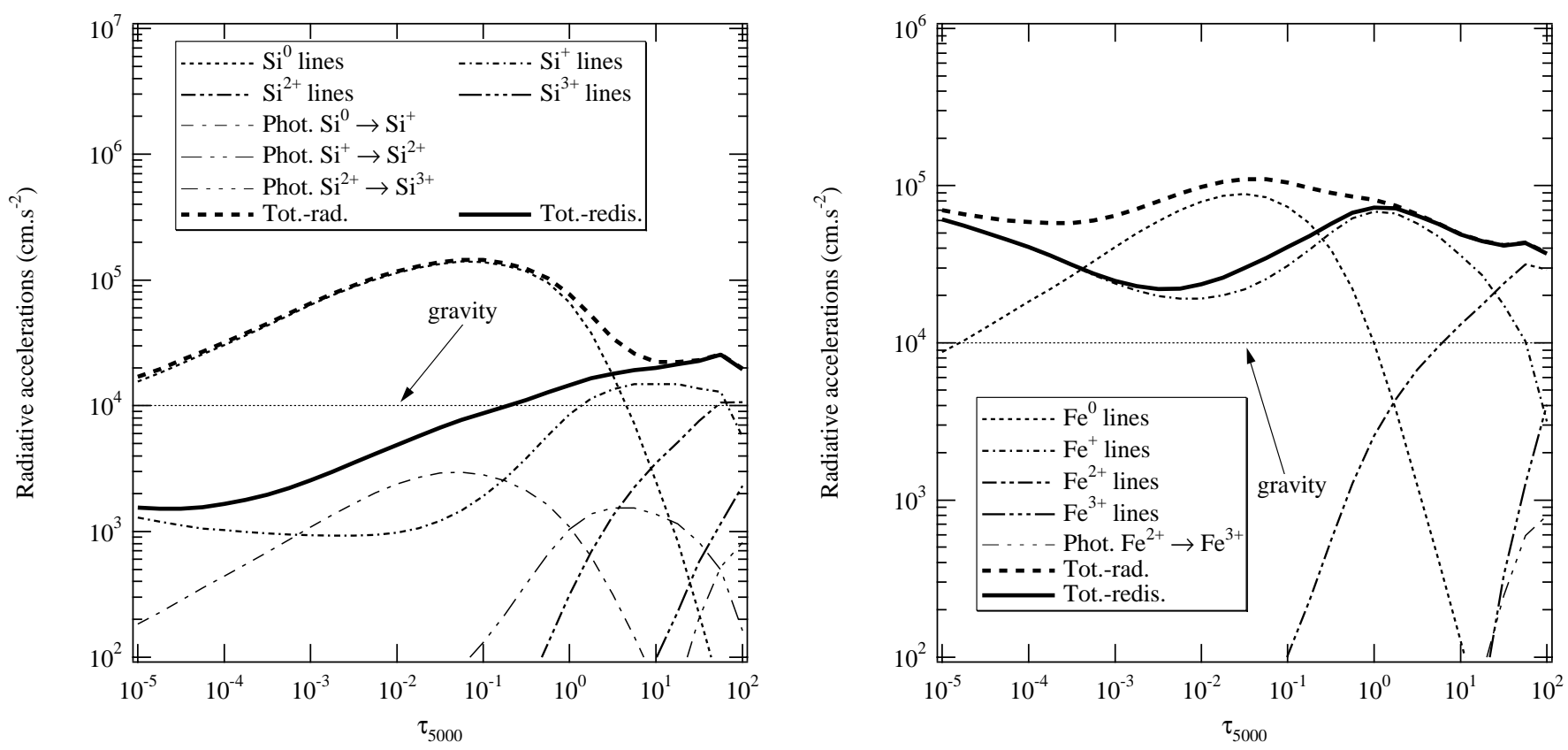

Fig. 3. Detail of the contributions of the various silicon (left panel) and iron (right panel) ions in a $T_{\text {eff }}=12000 \mathrm{~K}$, log $g=4$ model. The accelerations due to line absorptions are labelled "lines". The curves named "Phot." refer to the momentum acquired by photoionization. "Tot.-rad." and "Tot.-redis." show the behaviour of the total radiative acceleration respectively before and after application of the redistribution effect.
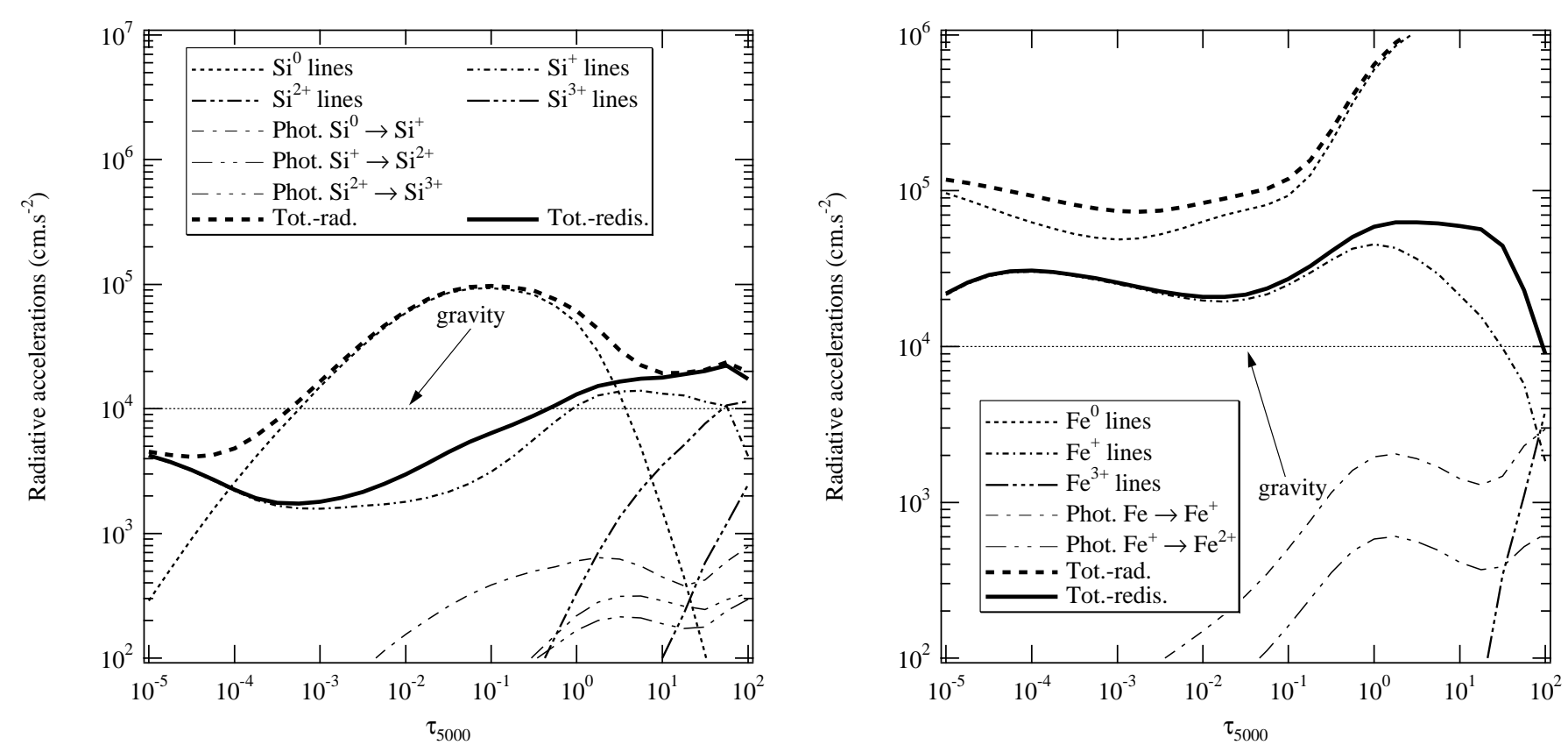

Fig. 4. Same as Fig. 3 with an NLTE calculation.

radiative accelerations dominated by the neutral contribution in the layers of concern (for instance, smaller effect on Co with respect to $\mathrm{Fe}$ and Ni since the contribution of the first ionization stage of Co dominates where redistribution is important). This is why redistribution is less efficient in the $18000 \mathrm{~K}$ model, for which the dominant contribution to the radiative accelerations is due to ionized stages. Also, redistribution is important if ionization rates are stronger than collisional rates. For most elements, this last condition is fulfilled when density is low enough (namely for $\tau_{5000} \leq 10^{-2}$ ). For CNO, though, redistribution is also significant in the deeper layers. In agreement with previous studies (GLAM, Hui-Bon-Hoa et al. 1996) the momentum transfer by recombination is negligible. For the $12000 \mathrm{~K}$ 
model, as the detail of the contributions of the individual ions are very similar for $\mathrm{Fe}, \mathrm{Ni}$, and $\mathrm{Zn}$, we can expect that redistribution could somewhat reduce the radiative accelerations for $\mathrm{Zn}$ - element for which there is no data for continuous absorption - around $\tau_{5000}=10^{-2}$, as it does for Fe and Ni. For a similar reason, the radiative accelerations of $\mathrm{Mn}, \mathrm{Cu}$, and $\mathrm{Ga}$ could be smaller too, by reference to Co.

Our results are in good overall agreement with previous studies on individual elements, despite different atomic data and computational methods. Let us remark that this agreement is achieved only if we take the redistribution effects into account. We will mainly compare here the results for the $12000 \mathrm{~K}$ model, since only one study has addressed the case of the $18000 \mathrm{~K}$ model (Alecian \& Michaud 1981 for $\mathrm{Mn}$ ). The major difference between this work and previous ones is the use of a NLTE model atmosphere and the NLTE computation of radiative accelerations in most of the studies (except Alecian \& Vauclair 1981, who used an Atlas 5 model). However, as the NLTE calculations are numerically very costly to carry out for the number of elements already studied, we limited ourselves to two cases (see Sect. 3.2), and we will discuss here our LTE results. The decrease of the Be radiative accelerations in the outer layers of Borsenberger et al. (1984) can originate from this NLTE treatment. Their relative population of BeII - the main contributor to the radiative accelerations - drops strongly as one goes upwards whereas ours remains important in the upper atmosphere. For boron, our total radiative accelerations are similar to those of Borsenberger et al. (1979), but we find that the main contribution between $\tau_{5000}=10^{-1}$ and $\tau_{5000}=10^{-5}$ comes from the neutral species, which they did not consider, this being compensated by radiative accelerations for BII almost ten times stronger than ours in these layers. The radiative accelerations for $\mathrm{Mg}$ is very near gravity in the line-forming region, as in Borsenberger et al. (1984). The decrease they get in the outer layers is certainly caused by a shift in the ionization balance which reduces the relative population of MgII, which is the main contributor to the radiative accelerations there. In the case of $\mathrm{Al}$, we find that the contribution of neutral $\mathrm{Al}$ is somewhat greater than in Hui-Bon-Hoa et al. (1996) around $\tau_{5000}=10^{-2}$, making the total radiative accelerations slightly stronger. This is due to a stronger relative population for Alı in our case. Let us mention that a mistake in the code used by Hui-Bon-Hoa et al. (1996) led to minor shifts in the optical depth scale (Alecian \& Hui-Bon-Hoa, private communication). As for silicon, the agreement is very good with Alecian \& Vauclair (1981). The radiative acceleration on CaII are close to those of Borsenberger et al. (1981), though, the total radiative accelerations we obtain are stronger in the upper atmosphere, mostly due to a different ionization balance.

Also, a similar reason causes the discrepancy between our radiative accelerations for $\mathrm{Mn}$ and those of Alecian \& Michaud (1981) in the outer layers, the values elsewhere being in very good agreement. For the $18000 \mathrm{~K}$ model, we have the same trend of stronger radiative accelerations with increasing temperature. As mentioned above, we have no atomic data for highly ionized gallium ions, making our radiative accelerations smaller than those of Alecian \& Artru (1987) where these ions have significant contributions, especially at the bottom of the atmosphere. Our GaII radiative accelerations are also slightly weaker, which is not surprising since different atomic data was used. The behaviour of the radiative accelerations for $\mathrm{Sr}$ is very similar to that found by Borsenberger et al. (1981), except in the superficial layers, where the shift of ionization balance when using a NLTE model could reduce the population of the singly ionized ion, which is the main contributor to the radiative accelerations. The same remark applies for Ba (Borsenberger et al. 1984).

At the bottom of the $12000 \mathrm{~K}$ atmosphere, we find a very good overall agreement with the radiative accelerations of the superficial layers of the $3 M_{\odot}$ inner structure model computed by Richer et al. (2000), as expected.

\subsection{NLTE calculations}

Since one of the purposes of this work is to test NLTE effects on the radiative accelerations, the calculations are performed using the same atmospheric structure as for the LTE computations, the elements we consider being silicon and iron. We detail the individual contributions for the Si and Fe ions in Fig. 4 for the $12000 \mathrm{~K}$ model.

The importance of NLTE effects depends on the species considered. For neutral silicon in the $12000 \mathrm{~K}$ model, the relative population vs. other ionization stages is lower than in LTE everywhere in the atmosphere, from about a factor of 10 at its bottom up to $10^{4}$ at $\tau_{5000} \approx 10^{-6}$. The changes in relative population and line radiative accelerations cancel out near the bottom of the atmosphere yielding similar line contributions but the NLTE line contributions are much smaller in the upper atmosphere. The deviations from LTE are very small for SiII except in the upper layers. The change in relative population is compensated by the line radiative accelerations so that the NLTE line contribution is close to that in LTE eveywhere in the atmosphere. For depths smaller than $\tau_{5000} \approx 10^{-4}$, the line contribution of neutral $\mathrm{Si}$ is much smaller than in LTE and SiII dominates the total radiative acceleration in the NLTE calculations. The net result is that the total radiative acceleration in this region is smaller than in LTE but when redistribution is considered, as the momentum transfer from Sir to SiII is very strong, the total redistributed radiative acceleration is still very near the line contribution of SiII so that its global behaviour is almost unchanged. The contribution of the radiative accelerations due to photoionization for $\mathrm{Si}$ is negligible for both LTE and NLTE calculations, but are much weaker in the NLTE case.

In the case of $\mathrm{Fe}$ in the $12000 \mathrm{~K}$ model, the behaviour of the ionization fractions is qualitatively the same as for $\mathrm{Si}$. A major difference is the change of the line radiative 

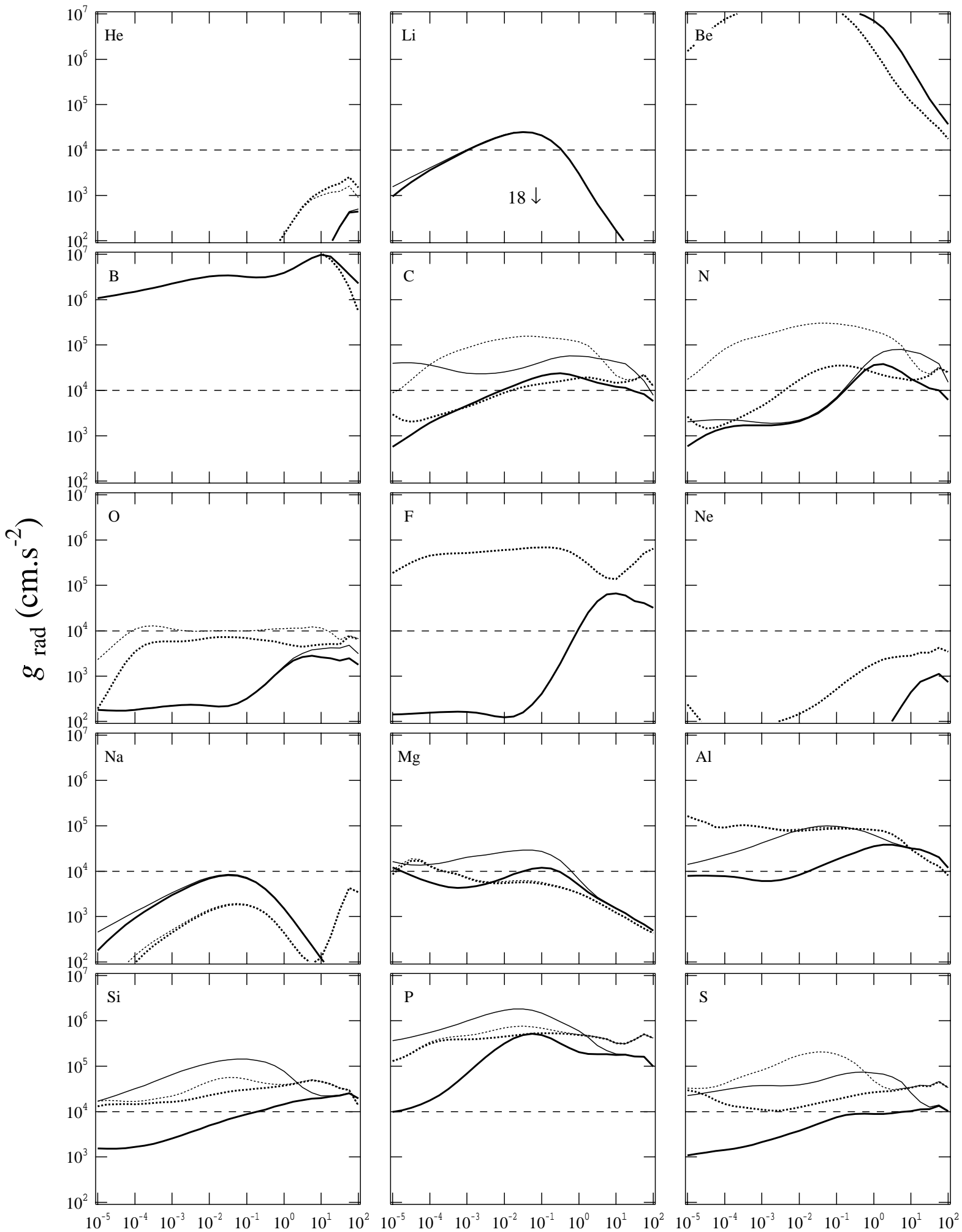

\section{$\tau_{5000}$}

Fig. 5. LTE radiative accelerations for the $12000 \mathrm{~K}$ and $18000 \mathrm{~K}$ models with $\log g=4$. The total radiative acceleration before (thin line) and after redistribution (thick line) are represented for a solar abundance of each element in the $12000 \mathrm{~K}$ model (solid lines), and in the $18000 \mathrm{~K}$ model (dotted lines). The long-dashed line denotes gravity. Downward arrows means that the radiative accelerations are lower than 100 for the corresponding models ( $T_{\text {eff }}$ next to the arrow, in $10^{3} \mathrm{~K}$, see text). The results for $(\mathrm{Cu}, \mathrm{Ga}, \mathrm{Rb}, \mathrm{Sr}, \mathrm{Y}, \mathrm{Zr}, \mathrm{Nb}, \mathrm{Ba}$, and $\mathrm{La})$ should be considered with caution at the bottom of the atmosphere since atomic data for certain ions are missing (see text for more details). 


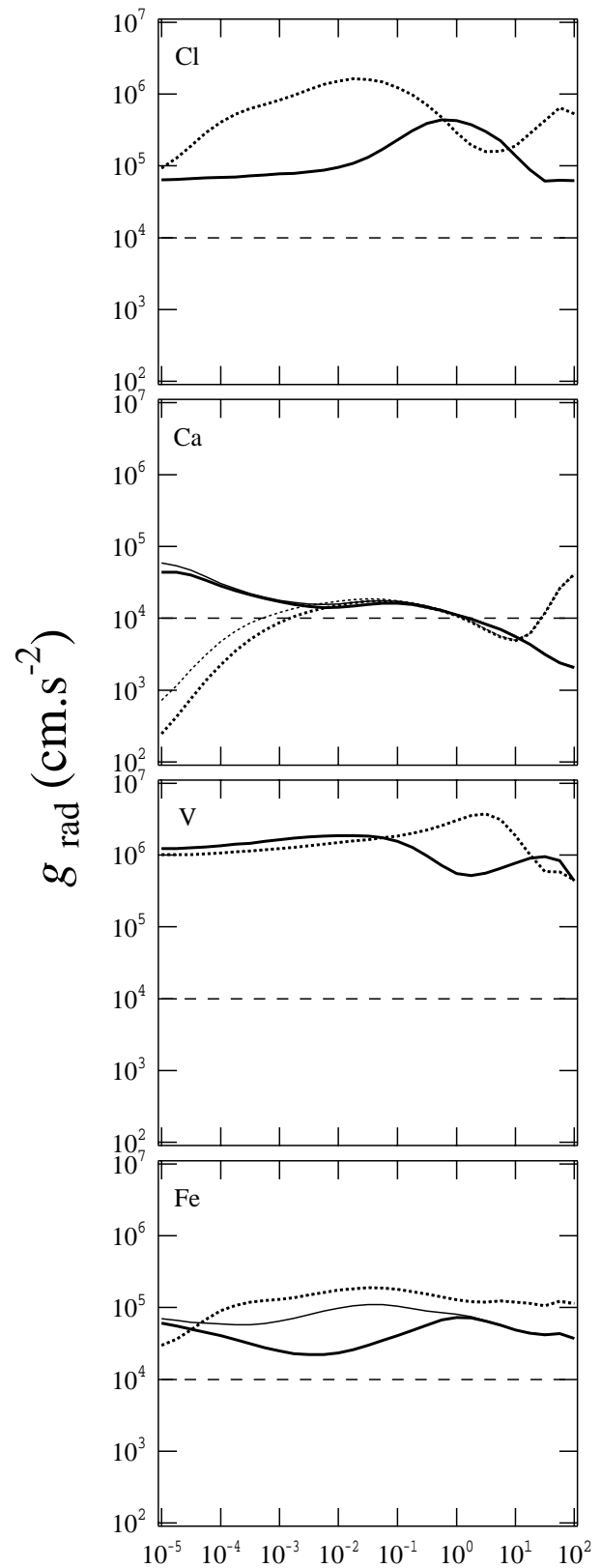

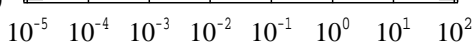
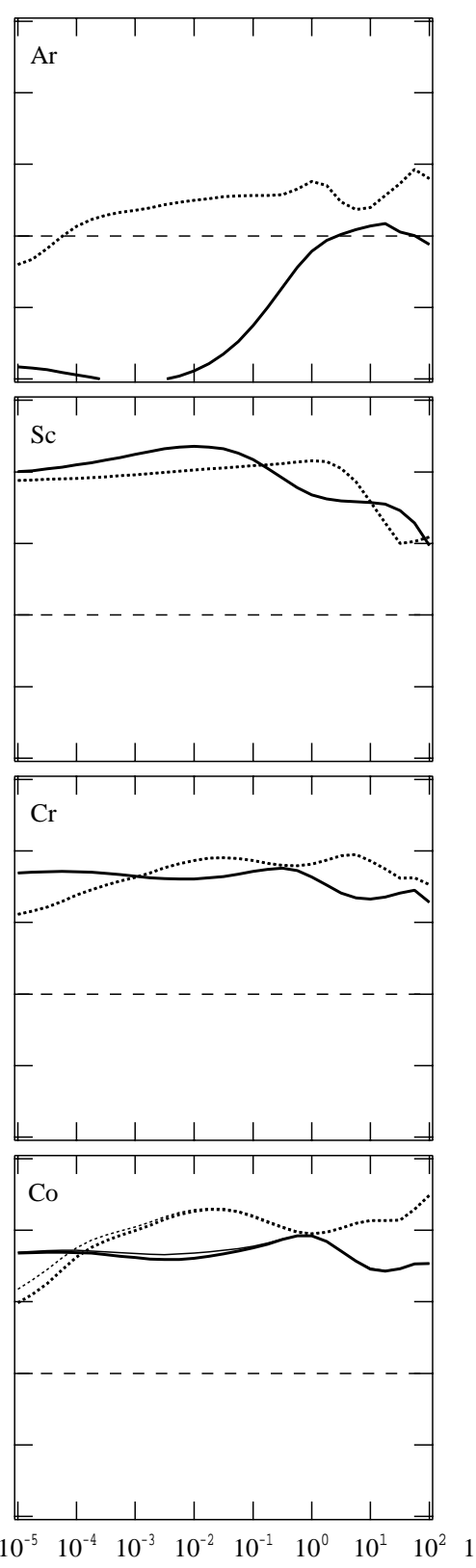

$\tau_{5000}$
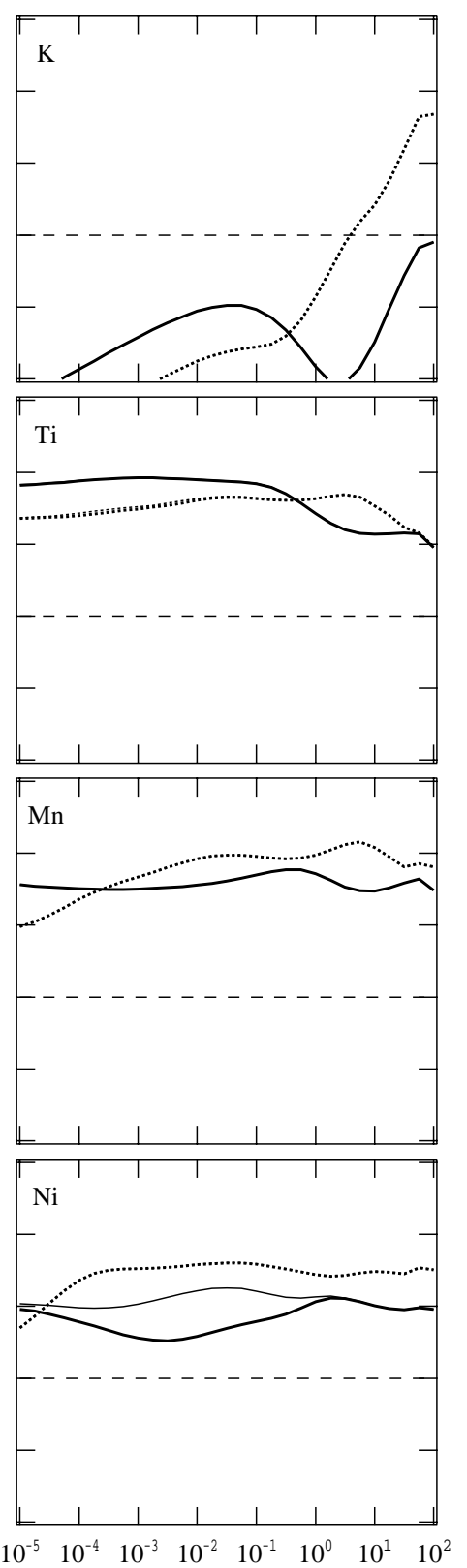

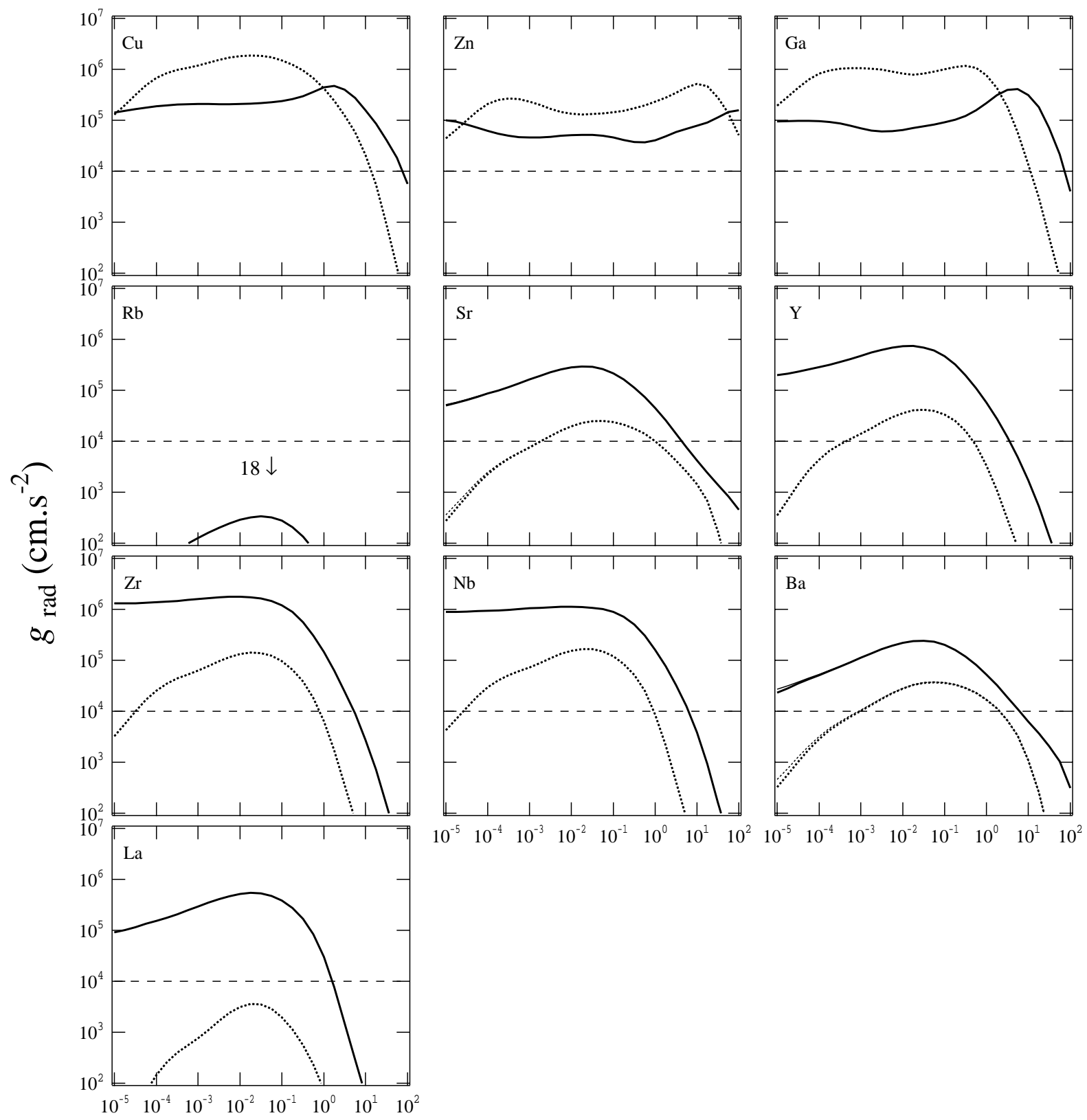

\section{$\tau_{5000}$}

Fig. 5. continued.

accelerations strongly enhance its contribution, which is dominant throughout the atmosphere. However, the total NLTE redistributed radiative accelerations are of the same strength as in LTE around $\tau_{5000} \approx 1$ because of a very strong redistribution effect. They are smaller at the bottom of the atmosphere and in the upper layers due also to redistribution, combined with an ionization shift towards FeII in these regions and to the weakness of the NLTE FeIII line radiative accelerations. Iron is then bound to the star since the total radiative accelerations are below gravity in the upper layers. As in the $12000 \mathrm{~K}$ model, the continuum radiative accelerations of FeII are much stronger in NLTE at the bottom of the atmosphere, making their contribution to the total radiative acceleration significant there.

\section{Comparison with observed anomalies}

The comparison between our results and the observed abundances is not straightforward. To make simulations of the abundance anomalies created by diffusion would require the computation of the time-dependent stratification process in the atmosphere and the amount of each element exchanged with the interior of the star (given by evolutionary models that take diffusion into account). Such calculations are beyond the scope of this study. However, 
as a first step, we can check if the radiative accelerations we compute are consistent with the observed anomalies.

In order to estimate the abundances supported by radiation in the atmosphere, we calculated the abundances of the various elements at equilibrium (i.e. $g_{\text {eff }}=0$ ). This procedure gives an estimation of the maximum abundance of the elements that can be supported by radiation in the atmosphere.

\section{1. $\mathrm{HgMn}$ stars}

The HgMn stars are non-magnetic CP stars whose $T_{\text {eff }}$ lie between 10000 and $15000 \mathrm{~K}$. The general abundance pattern has been depicted in the review paper of TakadaHidai (1991), to which we will refer mainly rather than individual studies since the abundance scatter is important among HgMn stars (e.g. Preston 1974). The observations are compared to the results of our $12000 \mathrm{~K}$ model. Since the radiative accelerations of $\mathrm{He}$ are smaller than gravity everywhere in the atmosphere, He settles and its content reduces as time goes by, which is consistent with the observed underabundances of this element. Li is slightly supported in the line-forming region and is bound to the star as the radiative accelerations are smaller than gravity in the upper layers. So, some accumulation could occur but no strong excesses are expected since the amount that could accumulate is small. Besides, the atmospheric content of $\mathrm{Li}$ should decrease with time since $\mathrm{Li}$ is not supported at the bottom of the atmosphere. This is in agreement with the fact that no strong overabundances of Li have been observed so far (Stephens \& Deliyannis 1999). Our calculations show that an overabundance of more than 5 dex of both $\mathrm{Be}$ and $\mathrm{B}$ can be supported by radiative forces in the line formation region. This is consistent with the large overabundances (up to approximately 4 dex) observed for Be and B (Takada-Hidai 1991). Slight overabundances (less than $1 \mathrm{dex}$ ) of $\mathrm{C}$ and small underabundances (approximately $-0.2 \mathrm{dex}$ ) of $\mathrm{N}$ can be produced in the line forming region. These values are greater than those observed, but as mentioned previously, exchanges of matter with the envelope during evolution could deplete these elements. According to our calculations, the maximum $\mathrm{O}$ abundance that can be supported is less than -0.5 dex if $\log g=4$. The observed abundance for this element in most $\mathrm{HgMn}$ stars is larger than this value and could be explained by a lower surface gravity, which would enhance the amount of $\mathrm{O}$ that can be supported. Finally, we find that the abundances of the iron-peak elements that can be supported in the atmosphere are consistent with those given by Takada-Hidai (1991).

\subsection{He-weak stars}

He-weak stars lie on the main sequence and have $T_{\text {eff }}$ approximately between 14000 and 20000 K. Since observational abundance determinations are much less prevalent for He-weak stars than for HgMn stars, we will only focus on the abundances of 3 Cen A given in Adelman \& Pintado (2000).

We find that in accordance to the observations, He is not supported in the atmosphere of the $T_{\text {eff }}=18000 \mathrm{~K}$ model and thus its abundance will be much smaller than its solar value. We also find that the maximum abundances of both CNO and the iron peak elements that can be supported by radiative pressure are greater than those observed and are then consistent with those given by Adelman \& Pintado (2000).

\section{Conclusion}

As expected, it was found that using the diffusion approximation for the radiative flux gives large errors for the radiative accelerations for optical depths smaller than unity. When studying diffusion in the atmosphere, it is imperative to solve explicitly the radiative transfer equation with a sufficiently fine wavelength sampling grid. NLTE effects can strongly modify the radiative accelerations of certain ions in stellar atmospheres. For the cases studied here, the difference between the total radiative acceleration using NLTE to those obtained using LTE was up to 0.5 dex in certain parts of the atmosphere. NLTE effects can also qualitatively change the behaviour of diffusion and should be considered when possible.

We also showed that for the vast majority of the elements considered here, the upper limits of the abundances caused by radiative diffusion in HgMn and He-weak stars are consistent with the observations. Our findings strongly support the belief that, contrary to Am stars (Richer et al. 2000) where diffusion is efficient only in the envelope, the abundance anomalies found in these stars are formed in the atmosphere.

Acknowledgements. This research was partially supported by a NSERC grant to the Université de Moncton and by NSF grants AST-9720704 and AST-0086246, NASA grants NAG5-8425, NAG5-9222, as well as NASA/JPL grant 961582 to the University of Georgia. This study made use of the computing resources of RQCHP at Université de Montréal, and of CNRS at IDRIS (project 011372). This work was also supported in part by the Pôle Scientifique de Modélisation Numérique at ENS-Lyon. Some of the calculations presented in this paper were performed on the IBM SP2 of the UGA UCNS, on the IBM SP "Blue Horizon" of the San Diego Supercomputer Center (SDSC), with support from the National Science Foundation, and on the IBM SP of the NERSC with support from the DoE. We thank all these institutions for a generous allocation of computer time. We thank G. Michaud for many interesting discussions and J. Richer for his help in the use of the computing resources at Université de Montréal. Thanks go also to the anonymous referee whose remarks helped improve the manuscript.

\section{References}

Adelman, S. J., \& Pintado, O. I. 2000, A\&A, 354, 899

Alecian, G. 1985, A\&A, 145, 275 
Alecian, G. 1994, A\&A, 289, 885

Alecian, G., \& Michaud, G. 1981, ApJ, 245, 226

Alecian, G., \& Vauclair, S. 1981, A\&A, 101, 16

Alecian, G., \& Artru, M.-C. 1987, A\&A, 186, 223

Alecian, G., \& Artru, M.-C. 1987, A\&A, 224, 323

Alecian, G., \& Vauclair, S. 1983, Fundamentals of Cosmic Physics (Gordon \& Breach Science Publishers Ltd.), 8, 369

Aller, L. H., \& Chapman, S. 1960, ApJ, 132, 461

Aufdenberg, J. P., Hauschildt, P. H., \& Baron, E. 1999, MNRAS, 302, 599

Aufdenberg, J. P., Hauschildt, P. H., Shore, S. N., \& Baron, E. 1998, ApJ, 498, 837

Barman, T. S., Hauschildt, P. H., Short, I. C., \& Baron, E. 2000, ApJ, 537, 946

Baron, E., \& Hauschildt, P. H. 1998, ApJ, 495, 370

Borsenberger, J., Praderie, F., \& Michaud, G. 1979, A\&A, 76, 287

Borsenberger, J., Praderie, F., \& Michaud, G. 1981, ApJ, 243, 533

Borsenberger, J., Praderie, F., \& Michaud, G. 1984, A\&A, 139, 147

Burgers, J. M. 1960, in Plasma Dynamics, ed. F. H. Clauser (Reading: Addison-Wesley), 119

Chapman, S., \& Cowling, T. G. 1970, The Mathematical Theory of Non-Uniform Gases, 3rd ed. (Cambridge Univ. Press, Cambridge)

CRC handbook of Phys. and Chem., 1997 (Cleveland, Ohio, CRC press)

Dreizler, S. 1999, in Reviews in Modern Astronomy, ed. R. E. Schielicke, 12, 255

Glaspey, J. W., Michaud, G., Moffat, A. F. J., \& Demers, S. 1989, ApJ, 339, 926

Gonzalez, J.-F., Artru, M.-C., \& Michaud, G. 1995a, A\&A, 302,788

Gonzalez, J.-F., LeBlanc, F., Artru, M.-C., \& Michaud, G. 1995b, A\&A, 297, 223 (GLAM)

Greenstein, G. S., Truran, J. W., \& Cameron, A. G. W. 1967, Nature, 213, 871

Greenstein, J. L., \& Sargent, A. I. 1974, ApJS, 28, 157

Grevesse, N., \& Noels, A. 1993, in La Formation des Eléments Chimiques, ed. B. Hauck, S. Paltani, \& D. Raboud (Lausanne, Switzerland), 205

Hauschildt, P. H., \& Baron, E. 1999, J. Comput. Applied Math., 102, 41

Hauschildt, P. H., Baron, E., Starrfield, S., \& Allard, F. 1996, ApJ, 462, 386
Hauschildt, P. H., Baron, E., \& Allard, F. 1997a, ApJ, 483, 390

Hauschildt, P. H., Shore, S., Schwartz, G. J., et al. 1997b, ApJ, 490, 803

Hauschildt, P. H., Allard, F., \& Baron, E. 1999, ApJ, 512, 377

Hui-Bon-Hoa, A., Alecian, G., \& Artru, M.-C. 1996, A\&A, 313, 624

Hui-Bon-Hoa, A., LeBlanc, F., \& Hauschildt, P. H. 2000, ApJ, 535, L43

Kurucz, R. L. 1994, Atomic Data for Opacity Calculations (Kurucz CD-ROM No. 1)

LeBlanc, F., \& Michaud, G. 1993, ApJ, 408, 251

LeBlanc, F., \& Michaud, G. 1995, A\&A, 303, 166

LeBlanc, F., Michaud, G., \& Richer, J. 2000, ApJ, 538, 876 (LMR2000)

Massacrier, G. 1996, A\&A, 309, 379

Massacrier, G., \& El-Murr, K. 1996, A\&A, 312, L25

Mathisen, R. 1984, Photo. Cross-sections for Stellar Atmosphere Calculations-Compilation of References and Data, Inst. Theor. Astrophys. Univ. Oslo, Publ. Ser. 1

Michaud, G. 1970, ApJ, 160, 641

Michaud, G., Vauclair, G., \& Vauclair, S. 1983, ApJ, 267, 256

Mihalas, D. 1978, Stellar Atmospheres, ed. W. H. Freeman (San Francisco)

Milne, E. A. 1927, M. N., 87, 697

Montmerle, T., \& Michaud, G. 1976, ApJS, 31, 489

Preston, G. W. 1974, ARA\&A, 12, 257

Reilman, R. F., \& Manson, S. T. 1979, ApJS, 40, 815

Richer, J., Michaud, G., \& Turcotte, S. 2000, ApJ, 529, 338

Richer, J., Michaud, G., \& Massacrier, G. 1997, A\&A, 317, 968

Richer, J., Michaud, G., Rogers, F., et al. 1998, ApJ, 492, 833

Schatzman, E. 1958, White Dwarfs (North-Holland Publ. Comp., Amsterdam)

Seaton, M. 1997, MNRAS, 289, 700

Sommerfeld, A. J. W., \& Schur, G. 1930, Atombau und Spektrallinien, vol. 2, 5th edition (Braunschweig: F. Vieweg)

Stephens, A., \& Deliyannis, C. P. 1999, PASP, 111, 482

Takada-Hidai, M. 1991, in Evolution of Stars: The Photospheric Abundance Connection, ed. G. Michaud, \& A. Tutukov (Kluwer Dordrecht), IAU Symp., 145, 137

Vauclair, G., Vauclair, S., \& Greenstein, J. L. 1979, A\&A, 80, 79 